\title{
Ultrasound fields in an attenuating medium
}

Jensen, Jørgen Arendt; Gandhi,, D; O'Brien,, W.D., Jr.

\section{Published in:}

Proceedings of the IEEE Ultrasonics Symposium

Link to article, DOI:

10.1109/ULTSYM.1993.339652

Publication date:

1993

Document Version

Publisher's PDF, also known as Version of record

Link back to DTU Orbit

Citation (APA):

Jensen, J. A., Gandhi, D., \& O'Brien, W. D. . J. (1993). Ultrasound fields in an attenuating medium. In Proceedings of the IEEE Ultrasonics Symposium (Vol. Volume 2, pp. 943-946). IEEE.

https://doi.org/10.1109/ULTSYM.1993.339652

\section{General rights}

Copyright and moral rights for the publications made accessible in the public portal are retained by the authors and/or other copyright owners and it is a condition of accessing publications that users recognise and abide by the legal requirements associated with these rights.

- Users may download and print one copy of any publication from the public portal for the purpose of private study or research.

- You may not further distribute the material or use it for any profit-making activity or commercial gain

- You may freely distribute the URL identifying the publication in the public portal

If you believe that this document breaches copyright please contact us providing details, and we will remove access to the work immediately and investigate your claim. 


\title{
ULTRASOUND FIELDS IN AN ATTENUATING MEDIUM
}

\author{
Jørgen A. Jensen ${ }^{1}$, Darshan Gandhi' ${ }^{2}$ and William D. O'Brien, Jr. ${ }^{2}$ \\ ${ }^{1}$ Electronics Institute, Build. 349, Technical University of Denmark, 2800 Lyngby, Denmark \\ ${ }^{2}$ Bioacoustics Research Laboratory, Department of Electrical and Computer Engineering, \\ University of Illinois at Urbana-Champaign, Urbana, IL 61801-2991, U.S.A.
}

\section{Abstract}

Ultrasound fields propagating in tissue will undergo changes in shape not only due to diffraction, but also due to the frequency dependent attenuation. Linear fields can be fairly well predicted for a non-attenuating medium like water by using the Tupholme- Stepanishen method for calculating the spatial impulse response, whereas the field cannot readily be found for an attenuating medium. In this paper we present a simulation program capable of calculating the field in a homogeneous attenuating medium. The program splits the aperture into rectangles and uses a far-field approximation for each of the rectangles and sums all contributions to arrive at the spatial impulse response for the aperture and field point. This approach makes it possible to model all transducer apertures, and the program can readily calculate the emitted, pulse-echo and continuous wave field. Attenuation is included by splitting it into a frequency dependent part and frequency independent part. The latter results in an attenuation factor that is multiplied onto the responses from the individual elements, and the frequency dependent part is handled by attenuating the basic one-dimensional pulse. The approach taken is very fast, and point spread functions can usually be generated within 10 seconds on commercially available workstations. The influence on ultrasound fields from attenuation is demonstrated.

\section{Introduction}

Modern medical ultrasound scanners use a variety of transducers. These include linear and convex arrays as well as annular arrays with advanced phasing strategies during both emission and reception of the ultrasound waves. Apodization of the individual transducer elements and over the whole aperture is employed in order to generate more homogeneous fields with diminished edge waves.
The optimization of the acoustic fields from the transducer is obtained by simulating and measuring the fields. The currently most efficient and accurate approach to the theoretical characterization of pulsed fields is that devised by Tupholme and Stepanishen [1], [2], [3]. Both the emitted and received field for the continuous and pulsed wave cases can be calculated and the theoretical model seems to accurately predict measured fields [4]. A fast simulation program has also been devised based on the Tupholme-Stepanishen approach [5]. The transducer is divided into squares and the far-field response from the individual squares added to yield the resulting field. This method makes it possible to simulate any transducer geometry, phasing, apodization and excitation.

The fields calculated by the above approach assumes propagation in a non-attenuating medium. Ultrasound propagating in human tissues experiences both absorption and scattering resulting in a frequency dependent attenuation. The transducers should, thus, be optimized for such a medium.

This paper introduces a program for calculating fields in a homogeneous attenuating medium, so various transducer geometries can be evaluated and optimized. The next section describes propagation in an attenuating medium and the improved simulation approach is detailed in Section 3. Results are presented in Section 4.

\section{Propagation in an attenuating medium}

The acoustic pressure field generated by an aperture mounted in an infinite, rigid baffle radiating into a homogeneous, non-attenuating medium is described by

$$
p(t, \vec{r})=\rho_{0} \frac{\partial v(t)}{\partial t} * h(t, \vec{r})
$$

where $\vec{r}$ denotes the position of the field point and $v(t)$ is particle velocity normal to the transducer surface. $\rho_{0}$ is 


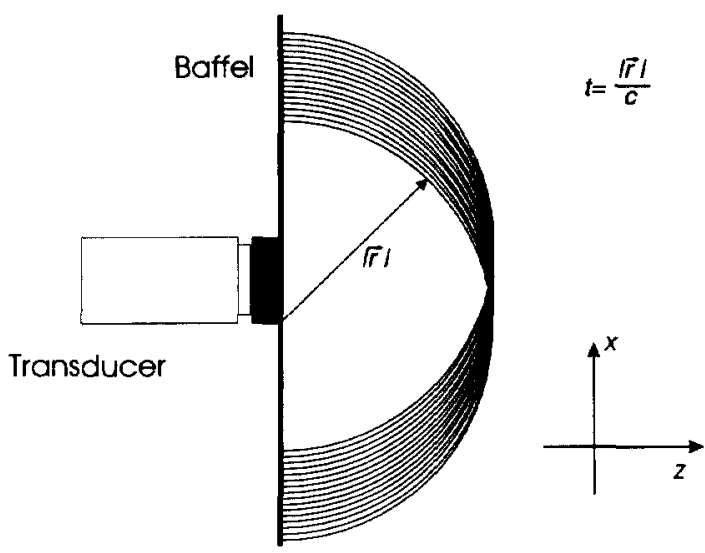

Figure 1: Summation of spherical waves from an aperture at a fixed time.

the density of the medium. $h(t, \vec{r})$ is the spatial impulse response and relates transducer geometry to the spatial extent of the field. It is calculated by the surface integral

$$
h(t, \vec{r})=\int_{S} \frac{\delta\left(t-\frac{\left|\vec{r}+\vec{r}_{1}\right|}{c}\right)}{\left|\vec{r}+\vec{r}_{1}\right|} d S
$$

where $S$ denotes the aperture and $\delta$ is the Dirac delta function. $\vec{r}_{1}$ denotes position over the aperture. The integral states that the field is found by integrating all spherical waves emanating from the transducer surface at the field point as shown in Fig. 1.

Submerging the transducer into a homogeneously attenuating medium will modify the propagation of the spherical waves, which will change continuously as a function of distance from the transducer. Eq. 2 is then changed"to

$$
h_{a t t}(t, \vec{r})=\int_{T} \int_{S} a\left(t-\tau,\left|\vec{r}+\vec{r}_{1}\right|\right) \frac{\delta\left(\tau-\frac{\left|\vec{r}+\vec{r}_{1}\right|}{c}\right)}{\left|\vec{r}+\vec{r}_{1}\right|} d S d \tau
$$

when linear propagation is assumed and the attenuation is the same throughout the medium. $a$ is the attenuation impulse response. The spherical wave is convolved with the distance dependent attenuation impulse response and spherical waves emanating from different parts of the aperture are convolved with different attenuation impulse responses.

A model for the attenuation must be introduced in order to solve the integral. Ultrasound propagating in tissue experiences a near linear with frequency attenuation and a popular attenuation amplitude transfer function is

$$
\left|A^{\prime}(f,|\vec{r}|)\right|=\exp \left(-\beta^{\prime} f \mid \vec{r} \eta\right)
$$

where $\beta^{\prime}$ is attenuation in nepers per meter. We here prefer to split the attenuation into a frequency depen- dent and a frequency independent part term as

$$
|A(f,|\vec{r}|)|=\exp (-\alpha|\vec{r}|) \exp \left(-\beta\left(f-f_{0}\right)|\vec{r}|\right)
$$

$\alpha$ is the frequency independent attenuation coefficient, and $f_{0}$ the transducer center frequency. The phase of the attenuation need also be considered. Kak and Dines (1978) introduced a linear with frequency phase response

$$
\Theta(f)=2 \pi f \tau_{b}|\vec{r}|
$$

where $\tau_{b}$ is the bulk propagation delay per unit length and is equal to $1 / c$. $c$ is the ultrasound propagation velocity. This, however, results in an attenuation impulse response that is non-causal. Gurumurthy and Arthur (1982) therefore suggested using a minimum phase impulse response, where the amplitude and phase spectrum form a Hilbert transform pair. The attenuation spectrum is then given by

$$
\begin{aligned}
A(f,|\vec{r}|) & =\exp (-\alpha \mid \vec{r}\rceil) \exp \left(-\beta\left(f-f_{0}\right)|\vec{r}|\right) \\
& \left.\times \exp \left(-j 2 \pi f\left(\tau_{b}+\tau_{m} \frac{\beta}{\pi^{2}}\right) \mid \vec{r}\right\rceil\right) \\
& \times \exp \left(j \frac{2 f}{\pi} \beta|\vec{r}| \ln (2 \pi f)\right)
\end{aligned}
$$

where $\tau_{m}$ is the minimum phase delay factor. Gurumurthy and Arthur (1982) suggests a $\tau_{m}$ value of 20 in order to fit the dispersion found in tissue.

The inverse Fourier transform of (7) must be inserted into (3) and the integral solved for the particular transducer geometry. This is clearly a difficult, if not impossible, task and some approximations must be introduced. All spherical wave arrives at nearly the same time instance, if the distance to the field point is very much larger than the transducer aperture. In this instance the attenuation function is, thus, the same for all waves and the result is a convolution between the attenuation impulse response and the spatial impulse response, which is a Dirac impulse. A spatial impulse response different from a Dirac function indicates that the spherical waves arrive at different times. Multiplying the arrival time with the propagation velocity gives the distance to the points on the aperture contributing to the response at that time instance. A first approximation is, therefore, to multiply the non-attenuated spatial impulse response with the proper frequency independent term. This approximation also assumes that the span of values for $\left|\vec{r}+\overrightarrow{r_{1}}\right|$ is so small that both $1 /\left|\vec{r}+\vec{r}_{1}\right|$ and the attenuation can be assumed to be roughly constant.

The frequency dependent function will also change for the different values of the spatial impulse response. A non-stationary convolution must, thus, be performed. One possible method to avoid this is to assume that the frequency dependent attenuation impulse response is 


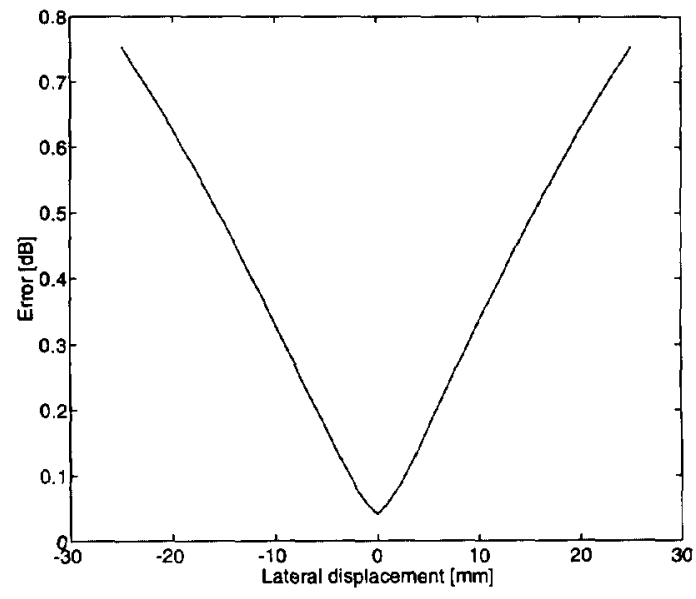

Figure 2: Error of assuming a non-varying frequency dependent attenuation over the duration of the spatial impulse response.

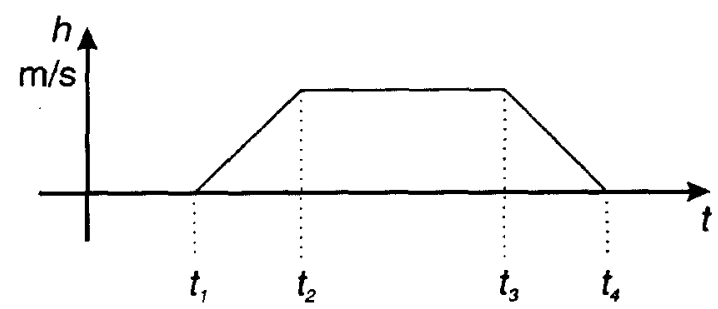

Figure 3: Far field response from a rectangular element

constant for the time and, thus, distances $\left|\vec{r}+\vec{r}_{1}\right|$ where $h$ is non-zero. The mean distance is then used in (7) and the inverse Fourier transform of $A\left(f,\left|\vec{r}_{m i d}\right|\right)$ is convolved with $h(t, \vec{r})$. The accuracy of the approach is dependent on the duration of $h$ and of the attenuation. The error in $\mathrm{dB}$ for a concave transducer with a radius of $10 \mathrm{~mm}$ focused at $100 \mathrm{~mm}$ and an attenuation of $0.5 \mathrm{~dB} /[\mathrm{MHz} \mathrm{cm}]$ is shown in Fig. 2. The axial distance to the transducer is $50 \mathrm{~mm}$.

\section{Simulation approach}

The simulation approach used is based on the program described in [5]. The transducer aperture is divided into small rectangles and the far-field spatial impulse response is calculated as a function of time for the particular point in space. The response has the shape of a trapezoid as shown in Fig. 3. The arrival times are determined by the distance from the four corners of the element to the field point.
The spatial impulse response has abrupt changes, when edges in the aperture are meet. This creates problems in a sampled system since a coarse sampling will result in a poor representation of the spatial impulse response. This can be alleviated by using a very high sampling frequency (in the $\mathrm{GHz}$ range), which then generates an unnecessarily high number of calculations. Another approach is to use the integrated spatial impulse response and after summation from all elements take the difference between adjacent sample values. This ensures that all the energy in the response is preserved and, thus, gives more accurate results. This is the method used here, and experience has shown that it even compares favorably to using the analytical expressions for the spatial impulse response for different apertures. The reason being is that this approach always preserves all the energy of an impulse response and that it is the total energy rather than the exact shape of the response that is important. The abrupt changes have spectral components that are higher than that of the transducer pulse and are, thus, of less importance.

The program calculates the spatial impulse response and the attenuation is introduced by multiplying the Fourier transformed of $h$ by the frequency dependent part of $(7) . \tau_{b}$ is set to zero, as the bulk delay is included in $h$. An inverse Fourier transform is performed on the result and the frequency independent attenuation is multiplied onto the response taking into account the actual distance to points on the aperture.

\section{Results}

The purpose of the program is to predict ultrasound fields and thereby make it possible to optimize the geometry, phasing, and apodization of an ultrasound transducer. The first example shows the optimization of a single element concave ultrasound transducer used for scanning cattle with the purpose of meat grading. The attenuation of the muscle tissue is between 0.5 to $0.7 \mathrm{~dB} /[\mathrm{MHz} \mathrm{cm}]$ and initially a $5 \mathrm{MHz}$ transducer with a radius of $12.7 \mathrm{~mm}$ and focused at $152.4 \mathrm{~mm}$ was used. The RMS pressure for such a transducer is shown in Fig. 4. The characteristic maximum of the pressure before the focus is seen in the non-attenuating medium. Introducing an attenuation of $0.7 \mathrm{~dB} /[\mathrm{MHz} \mathrm{cm}]$ significantly reduces the received signal. A low returned signal is experienced from the depth from 4 to $12 \mathrm{~cm}$, where the longissimus muscle is located. Selecting a shorter focus of $102 \mathrm{~mm}$ and changing the transducer frequency to $3 \mathrm{MHz}$ result in a substantially higher RMS pressure that remains roughly constant within the region of interest.

The second example shows the influence of attenuation on the point spread function (PSF). A concave 


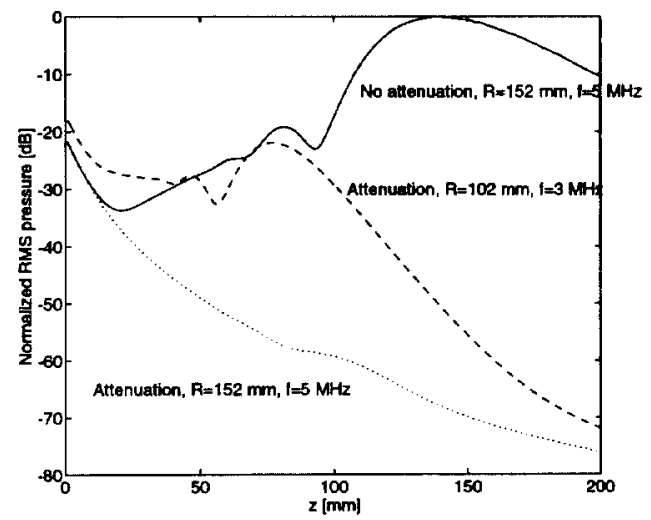

Figure 4: RMS pulsed pressure on the acoustical axis received by different transducers submerged in different media.

transducer with a radius of $8 \mathrm{~mm}$, center frequency of $3 \mathrm{MHz}$, and focused at $100 \mathrm{~mm}$ was used. Fig. 5 shows point spread functions calculated under different conditions. The logarithmic envelope of the received pressure is displayed as a function of time and lateral displacement. The left most graph shows the normalized PSF for the transducer submerged in a non-attenuating medium. The distance to the field point is $60 \mathrm{~mm}$ and the function is shown for lateral displacements from -8 to $8 \mathrm{~mm}$. Introducing a $0.5 \mathrm{~dB} /[\mathrm{MHz} \mathrm{cm}]$ attenuation yields the normalized PSF shown in the middle. The central core of the PSF does not change significantly, but the shape at $-30 \mathrm{~dB}$ and below are somewhat different from the non-attenuated response. A slightly broader and longer function is seen, but the overall shape is the same.

An often used approach to characterize the field is to include the attenuation into the basic one-dimensional pulse, and then use the non-attenuated spatial impulse response in calculating the PSF. This is the approach used in the rightmost graph in Fig. 5. All attenuation is included in the pulse and the spatial impulse response calculated in the leftmost graph is used for making the PSF. The similarity to the center graph is striking. Apart from a slightly longer response, nearly all features of the field are the same. It is, thus, appropriate to estimate the attenuated one-dimensional pulse and reconstruct the whole field from this and knowledge of the transducer geometry.

\section{References}

[1] G. E. Tupholme. Generation of acoustic pulses by baffled plane pistons. Mathematika, 16:209-224,
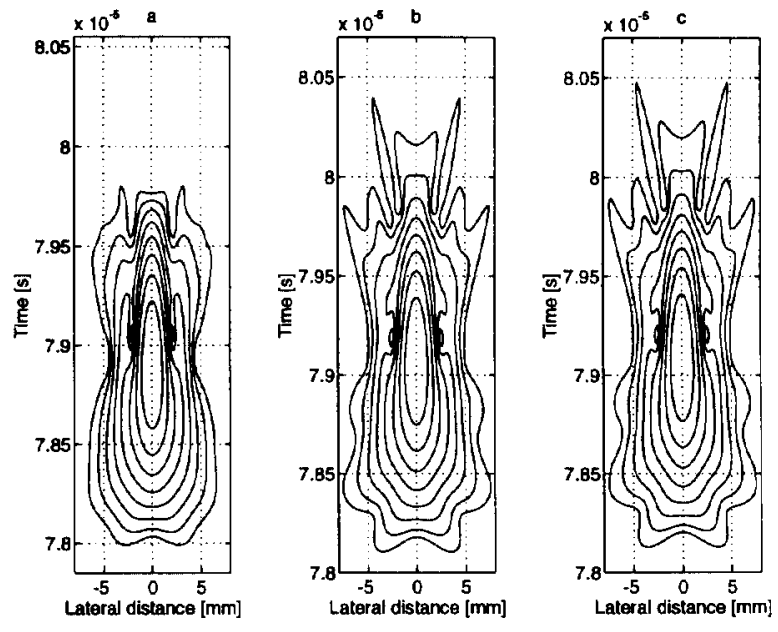

Figure 5: Contour plots of point spread functions for different media and calculation methods. a: Non attenuating medium. b: $0.5 \mathrm{~dB} /[\mathrm{MHz} \mathrm{cm}]$ attenuation. $\mathrm{c}: 0.5$ $\mathrm{dB} /[\mathrm{MHz} \mathrm{cm}]$ attenuation on the one-dimensional pulse. There is $6 \mathrm{~dB}$ between the contour lines. The distance to the transducer is $60 \mathrm{~mm}$.

1969.

[2] P. R. Stepanishen. The time-dependent force and radiation impedance on a piston in a rigid infinite planar baffle. Journal of the Acoustical Society of America, 49:841-849, 1971.

[3] P. R. Stepanishen. Transient radiation from pistons in an infinte planar baffle. Journal of the Acoustical Society of America, 49:1629-1638, 1971.

[4] J. A. Jensen. A model for the propagation and scattering of ultrasound in tissue. Journal of the Acoustical Society of America, 89:182-191, 1991.

[5] J. A. Jensen and N. B. Svendsen. Calculation of pressure fields from arbitrarily shaped, apodized, and excited ultrasound transducers. IEEE Transactions Ultrasonics, Ferroelectrics, and Frequency Control, 39:262-267, 1992.

[6] A. C. Kak and K. A. Dines. Signal processing of broadband pulse ultrasound: measurement of attenuation of soft biological tissues. IEEE Transactions on Biomedical Engineering, BME-25:321-344, 1978.

[7] K. V. Gurumurthy and R. M. Arthur. A dispersive model for the propagation of ultrasound in soft tissue. Ultrasonic Imaging, 4:355-377, 1982. 